\title{
Pleno empleo: Concepto y estimaciones para las regiones españolas
}

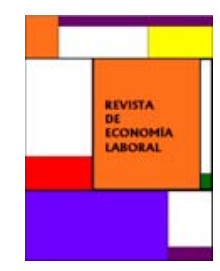

\author{
Javier Capó Parrilla * y Francisco Gómez García ${ }^{\dagger, 1}$
}

* Universidad de las Islas Baleares y CRE. † Universidad de Sevilla

Recibido el 20 de septiembre de 2005; aceptado el 1 de marzo de 2006

Received 20 September 2005; accepted 1 March 2006

\section{Resumen:}

En este trabajo estimamos el pleno empleo de cada una de las regiones españolas. Para atender a dicha finalidad utilizamos distintas metodologías, lo que nos permite realizar comparaciones: a) Métodos de alisamiento; b) Equilibrio a largo plazo (solución particular de un AR(1) para la tasa de desempleo); y c) El enfoque de los salarios (estimación de "time-varying" NAWRUs utilizando el método de Elmeskov).

Palabras clave: Pleno empleo, NAWRU, filtro de Hodrick-Prescott, mercados de trabajo regionales.

Clasificación JEL: R23, J23, J60.

\begin{abstract}
:
In this article we estimate full employment for the Spanish regions. To do that we implement and compare some alternative methodologies: a) Smoothing methods; b) Longterm equilibrium (particular solution of an AR(1) for unemployment); and c) The wage approach (estimation of time-varying NAWRUs with Elmeskov method).
\end{abstract}

Keywords: Full employment, NAWRU, Hodrick-Prescott filter, regional labor markets. JEL Classification: R23, J23, J60.

\footnotetext{
1 Autor para correspondencia: fgomez@us.es. Este trabajo fue presentado en las VI Jornadas de Economía Laboral (Alicante, 2005) y una versión del mismo ha sido aceptada para su publicación en la serie de Papeles de Trabajo del Instituto de Estudios Fiscales (18/2005). Agradecemos los comentarios sobre un borrador inicial de Roberto Bande y Fernando Núñez. Asimismo, agradecemos las sugerencias de un evaluador anónimo y del consejo de redacción de la Revista de Economía Laboral. Los errores que persistan son de nuestra exclusiva responsabilidad.
}

(C) Revista de Economía Laboral 


\section{Introducción}

La Cumbre Europea de Lisboa (2000) señaló, entre otras cuestiones, los fuertes desequilibrios regionales existentes en Europa en materia de desempleo. Sin embargo, en la misma se decidió plantear los objetivos para el mercado de trabajo no en función del nivel de desempleo, sino en función del nivel de empleo: se pretende que el 70\% de la población adulta (16-65 años) tenga empleo en el año 2010. No obstante, tanto desde un punto de vista teórico como político, el pleno empleo se ha relacionado con una tasa de paro reducida, distinta para cada economía.

Aunque en los últimos años la situación ha mejorado, el deficiente funcionamiento del mercado de trabajo sigue siendo uno de los rasgos más preocupantes de la economía española. Por ello, en este trabajo se estima la tasa de paro asociada al pleno empleo de cada una de las regiones españolas. Para atender a dicha finalidad se utilizan distintas metodologías, lo que permite realizar comparaciones interregionales: a) Métodos de alisamiento; b) Equilibrio a largo plazo (solución particular de un $\mathrm{AR}(1)$ para la tasa de desempleo); y c) El enfoque de los salarios (estimación de "time-varying" NAWRUs utilizando el método de Elmeskov).

La estructura del trabajo es la siguiente. En el apartado segundo se delimita conceptualmente el concepto de "pleno empleo". En el apartado tercero se implementan distintas metodologías para estimar dicho inobservable para las Comunidades Autónomas españolas. Por último, en el apartado cuarto se recogen algunas reflexiones finales y se plantean algunas posibles extensiones del análisis.

\section{Pleno Empleo: Delimitación Conceptual}

La expresión "pleno empleo" empezó a utilizarse de forma general en la economía después de la depresión de los años treinta. Se aplicó a aquellas economías industrializadas en las que la inmensa mayoría de la población activa se encontraba empleada. Pero esta definición del pleno empleo es demasiado imprecisa y ambigua, tanto desde el punto de vista conceptual como estadístico. A continuación, se realiza una breve revisión de los principales intentos de definición del concepto de pleno empleo, lo que puede ser un punto de partida útil para informar y evaluar las metodologías que se proponen a continuación.

En primer lugar, los economistas clásicos, a los que se oponía Keynes, basándose en la noción de equilibrio del mercado de trabajo, 
creyeron que el pleno empleo era aquel volumen de empleo hacia el que tendía la economía. Lograr el pleno empleo, en ese contexto teórico, exigía de los gobiernos nada más y nada menos que la neutralidad.

Una vez que Keynes (1936) puso en tela de juicio dicha noción clásica, el pleno empleo vino a significar la oferta máxima global, el punto en el cual la expansión de la demanda agregada no podía aumentar el empleo y la producción. En este contexto, el pleno empleo fue también considerado como el umbral de inflación de la economía -teoría del "gap" inflacionista-.

Una definición de pleno empleo, asociada a Beveridge (1944), es que el número de desempleados iguale al número de vacantes no cubiertas -curva UV- (en realidad, Beveridge exigió en su concepto de pleno empleo un exceso de vacantes no cubiertas sobre los desempleados). En este caso, el desempleo de equilibrio se aproxima por la suma del desempleo friccional y estructural. El problema de esta medida es su difícil cuantificación, ya que no es fácil obtener estimaciones fiables del número de vacantes no cubiertas.

Por otro lado, también son de destacar las definiciones basadas en la experiencia histórica, como la que se obtiene del enfoque del desempleo mínimo. Dicho enfoque dice que existe pleno empleo cuando el nivel de desempleo es igual al valor más bajo alcanzado en el último ciclo. Este tipo de definición tiene el problema de que, debido a las circunstancias cambiantes, el "mínimo irreducible" de un periodo anterior puede diferir sustancialmente del mínimo actual (esto parece evidente para la economía española, como señalamos a continuación). Muchas veces se afirma que técnicamente el pleno empleo está asociado a una tasa de desempleo del $5 \%$. Sin embargo, este tipo de mediciones descansan en un supuesto ad hoc.

Por último, está el enfoque de los precios, según el cual si el desempleo cae por debajo de un cierto nivel, el resultado es necesariamente un incremento de los precios. Así pues, el pleno empleo podría definirse como una situación caracterizada por un nivel de desempleo compatible con un nivel de precios estable.

Desde esta última perspectiva no puede obviarse la pregunta de si la Tasa Natural de Desempleo es otro candidato al concepto de pleno empleo. Friedman (1968) definió la tasa natural como aquella tasa de desempleo que es consistente con el equilibrio en un sistema walrasiano, rectificado de manera que se tengan en cuenta: 1) las características estructurales de los mercados de bienes y de trabajo; 2) las variaciones estocásticas en las demandas y las ofertas en dichos mercados; y 3) los costes de obtención de la información y los costes de la movilidad. Puede apreciarse la inclusión del desempleo friccional en la definición de 
Pleno empleo: Concepto y estimaciones para las regiones españolas

Friedman. Por otro lado, también se pone el énfasis en las características estructurales de los mercados de trabajo y de bienes, junto a las variaciones de las ofertas y las demandas respectivas. Ello, en presencia de costes de movilidad, genera desempleo estructural, tanto en su vertiente ocupacional como geográfica. ${ }^{2}$

\section{Metodologías para Estimar el Pleno Empleo. Aplicación al Caso de las Regiones Españolas}

En este apartado se obtiene la tasa de paro de equilibrio, como aproximación del pleno empleo, de las regiones españolas en el periodo 1990-2004. A partir de los datos de la tasa de paro trimestral procedentes de la Encuesta de Población Activa (EPA) se han aplicado diversas metodologías para estimar estadística y econométricamente esta variable inobservable.

\subsection{Métodos de Alisamiento}

Se trata de un método de estimación del pleno empleo muy difundido, que puede presentar distintas variantes más o menos complejas. Así, por ejemplo, está el cálculo de la media de las tasas de desempleo observadas a lo largo de un periodo amplio de tiempo o el cálculo de medias móviles. Por otro lado, se puede utilizar como punto de referencia periodos en los que se piense que el mercado de trabajo se encuentra en relativo equilibrio (próximo al pleno empleo). Otros métodos son el ajuste de tendencias ${ }^{3}$ y la aplicación del filtro de Hodrick-Prescott.

La principal ventaja de este método de estimación radica en su sencillez, ya que básicamente la única información necesaria consiste en los valores pasados del desempleo observado, a los que se aplican técnicas de análisis de series temporales de tipo univariante. En cuanto a sus limitaciones, hay que señalar la ausencia de sustrato teórico, que no se consideran en el análisis variables adicionales -aparte del desempleo observado- que podrían proporcionar más información, y que tampoco se

2 Una constatación llamativa de la ambigüedad del concepto Tasa Natural la encontramos en el trabajo de Rogerson (1997), donde se encuentran hasta once versiones de dicho concepto: tasa de desempleo de largo plazo, friccional, media, de equilibrio, normal, pleno empleo, necesaria, de estado estacionario, mínima sostenible, eficiente, tendencia de Hodrick-Prescott. Por supuesto, a esta lista habría que añadir la de NAIRU y NAWRU.

3 Para una valoración de las estimaciones basadas en la tendencia pueden consultarse los trabajos de Staiger et al. (1997) y Staiger et al. (2001). 
apuntan los factores determinantes del pleno empleo. ${ }^{4}$

El cuadro 1 recoge los resultados obtenidos de aplicar el filtro de Hodrick y Prescott a las tasas de paro de las regiones españolas en el periodo 1990-2004. Para tener en cuenta los distintos cambios metodológicos acaecidos en la EPA se ha utilizado la serie homogénea que publica el INE. Como era de esperar, el filtro ${ }^{5}$ alisa la serie original de paro, pero se hace necesario aportar más información adicional. A dicha tarea se dedican las siguientes secciones del trabajo.

\section{Cuadro 1: Medias anuales de las tasas de paro trimestrales alisadas con el filtro de Hodrick-Prescott para las regiones españolas (1990-2004)}

\begin{tabular}{|c|c|c|c|c|c|c|c|c|c|c|c|c|c|c|c|}
\hline & 90 & 91 & 92 & 93 & 94 & 95 & 96 & 97 & 98 & 99 & 00 & 01 & 02 & 03 & 04 \\
\hline Andalucía & 25,2 & 27,4 & 29,5 & 31,5 & 32,7 & 33,0 & 32,3 & 30,9 & 28,8 & 26,4 & 23,9 & 21,6 & 19,7 & 18,1 & 16,5 \\
\hline Aragón & 9,7 & 11,7 & 13,6 & 15,1 & 16,0 & 15,9 & 15,0 & 13,5 & 11,6 & 9,7 & 8,0 & 6,8 & 6,0 & 5,5 & 5,1 \\
\hline Asturias & 16,5 & 17,4 & 18,4 & 19,6 & 20,5 & 20,8 & 20,7 & 19,9 & 18,6 & 16,8 & 19,7 & 12,7 & 11,2 & 10,1 & 9,2 \\
\hline Baleares & 10,5 & 12,0 & 13,5 & 14,7 & 15,1 & 14,6 & 13,5 & 12,0 & 10,5 & 9,0 & 8,0 & 7,6 & 7,7 & 8,1 & 8,6 \\
\hline Canarias & 24,1 & 25,0 & 25,6 & 25,7 & 25,1 & 23,8 & 22,0 & 19,6 & 17,6 & 15,4 & 13,6 & 12,3 & 11,5 & 11,1 & 10,8 \\
\hline Cantabria & 15,9 & 17,3 & 18,8 & 20,3 & 21,4 & 21,9 & 21,4 & 20,0 & 18,0 & 15,7 & 13,5 & 11,7 & 10,6 & 9,9 & 9,4 \\
\hline Cataluña & 12,1 & 13,9 & 15,9 & 17,6 & 18,7 & 18,8 & 18,0 & 16,4 & 14,4 & 12,2 & 10,8 & 9,8 & 9,4 & 9,3 & 9,2 \\
\hline Castilla y León & 15,3 & 16,7 & 18,2 & 19,5 & 20,3 & 20,4 & 19,9 & 18,9 & 17,3 & 15,6 & 13,8 & 12,3 & 11,2 & 10,4 & 9,8 \\
\hline Cast-La Mancha & 13,0 & 14,8 & 16,5 & 18,1 & 19,1 & 14,9 & 19,0 & 18,3 & 16,5 & 14,7 & 13,0 & 11,4 & 10,2 & 9,4 & 8,8 \\
\hline Extremadura & 24,3 & 25,8 & 27,4 & 28,9 & 30,0 & 30,4 & 30,0 & 28,9 & 27,2 & 24,9 & 22,5 & 20,2 & 18,5 & 17,2 & 16,1 \\
\hline Galicia & 12,4 & 14,2 & 15,9 & 17,4 & 18,4 & 18,7 & 18,6 & 18,0 & 17,0 & 15,8 & 14,6 & 13,5 & 12,8 & 12,5 & 12,4 \\
\hline C. Madrid & 11,4 & 13,3 & 15,3 & 17,2 & 18,7 & 19,3 & 19,0 & 17,9 & 16,0 & 13,8 & 11,6 & 9,6 & 8,1 & 6,9 & 5,8 \\
\hline Murcia & 17,0 & 19,4 & 21,5 & 23,1 & 23,7 & 23,3 & 21,9 & 19,9 & 17,5 & 15,3 & 13,4 & 12,0 & 11,1 & 10,4 & 9,9 \\
\hline Navarra & 11,4 & 11,9 & 12,5 & 13,0 & 13,1 & 12,6 & 11,8 & 10,6 & 9,4 & 8,0 & 6,9 & 6,0 & 5,6 & 5,3 & 5,1 \\
\hline País Vasco & 18,5 & 19,8 & 21,0 & 21,9 & 22,3 & 21,8 & 20,5 & 18,7 & 16,7 & 14,6 & 12,6 & 11,1 & 10,0 & 9,3 & 8,7 \\
\hline La Rioja & 9,1 & 11,1 & 12,9 & 14,3 & 15,0 & 14,8 & 13,9 & 12,4 & 10,8 & 9,2 & 7,9 & 6,8 & 6,1 & 5,6 & 5,1 \\
\hline C. Valenciana & 15,0 & 17,4 & 19,7 & 21,5 & 22,4 & 22,2 & 21,0 & 19,1 & 16,9 & 14,6 & 12,7 & 11,4 & 10,6 & 10,2 & 9,9 \\
\hline
\end{tabular}

Fuente: Elaboración propia a partir de los datos de la EPA (serie homogénea del INE, 1990-2004).

\subsection{Equilibrio a Largo Plazo}

Podemos esperar que las regiones que se ajustan lentamente ante los shocks de demanda tendrán una tasa de desempleo de pleno empleo mayor que el de las regiones en que dicho ajuste es más rápido. Este grado de persistencia puede ser medido estimando un modelo univariante para sus series temporales de desempleo -se puede encontrar un ejemplo en el trabajo de Pehkonen y Turvo (1998) para las regiones de Finlandia-. Para

$4 \mathrm{El}$ comentario anterior puede matizarse notablemente si se hace referencia a la aplicación del filtro de Kalman o del filtro de Hodrick-Prescott multivariante, métodos muy utilizados recientemente en las estimaciones de la NAIRU. Una buena muestra la proporciona Richardson et al. (2000).

${ }^{5}$ El filtro se aplica inicialmente a la serie trimestral de desempleo y posteriormente se calcula la media anual. 
ello se utiliza el siguiente modelo de regresión:

$$
U_{t}=\alpha+\beta U_{t-1}+\varepsilon_{t}
$$

donde $U$ es la tasa de desempleo trimestral desesestacionalizada, t es el tiempo y $\alpha$ y $\beta$ son coeficientes. Se espera que el coeficiente $\beta$ esté entre 0 y 1 (ésto garantiza la monotonía y la estabilidad del modelo). Un valor alto de $\beta$ indica que la tasa de desempleo actual tiene una alta correlación con la tasa de desempleo del periodo anterior. Un valor bajo de $\beta$ indica que la tasa de desempleo actual está muy poco correlacionada con la tasa de desempleo del periodo anterior (de hecho, si $\beta=0$ entonces la tasa de desempleo fluctuará aleatoriamente en torno a su media, que en este caso será $\alpha$, pues se asume que el término de error está distribuido normalmente con un valor medio igual a cero -ruido blanco-).

Una vez que los parámetros de la ecuación [1] han sido estimados, la tasa de desempleo de pleno empleo (desempleo de equilibrio a largo plazo) puede ser calculada para cada región. Si se interpreta que el equilibrio está asociado a una tasa de desempleo constante, es decir,

$$
U_{t}=U_{t-1}=U^{*}
$$

donde $U^{*}$ es la tasa de desempleo de equilibrio y asumiendo que el término de error es cero en el largo plazo. Por tanto, obteniendo la solución particular de la ecuación [1]:

$$
U^{*}=\frac{\alpha}{1-\beta}
$$

El supuesto implícito en la ecuación [3] es que la tasa de paro se mantiene estable en el tiempo. Esta circunstancia sólo es aceptable, en la economía española, para el periodo 2001-2004 ya que en el periodo anterior, entre 1990 y 1994, se observa un aumento de la tasa de paro y, a continuación, entre 1995 y 2001, se produce un descenso sostenido y generalizado de las tasas de paro. Por ello, únicamente se ha aplicado esta metodología al subperiodo 2001-2004. ${ }^{6}$

$\mathrm{Al}$ realizar las estimaciones se ha contrastado la existencia de autocorrelación a través del test de Breusch y Godfrey, basado en el multiplicador de Lagrange, y en caso de detectar la existencia de autocorrelación se ha introducido como regresores más retardos de la variable endógena, contrastando posteriormente la significación conjunta de los parámetros $\beta$ mediante el test de Wald. En este caso particular, la tasa de paro de equilibrio U* adopta la siguiente expresión:

\footnotetext{
6 Se ha corregido la estacionalidad de las tasas de paro trimestrales mediante la aplicación del Método Census X11 Multiplicativo.
} 


$$
U^{*}=\frac{\alpha}{1-\sum \beta}
$$

El cuadro 2 recoge los resultados de la regresión de la ecuación [1] y el cuadro 3 la tasa de paro de equilibrio que se deriva de la ecuación [3] en aquellos casos en que los parámetros han sido significativos.

\section{Cuadro 2: Estimaciones de un modelo univariante para las tasas de desempleo regionales en España}

\begin{tabular}{|c|c|c|c|c|}
\hline \multicolumn{5}{|c|}{ Variable dependiente: tasa de paro trimestral desestacionalizada $\left(\mathrm{U}_{\mathrm{t}}\right)$} \\
\hline \multicolumn{5}{|c|}{ Método de estimación: mínimos cuadrados ordinarios (MCO) } \\
\hline \multicolumn{5}{|c|}{ Periodo muestral: 2001:2 -2004:4 } \\
\hline \multicolumn{5}{|c|}{ Número de regiones: 17} \\
\hline \multicolumn{5}{|c|}{ Número de observaciones: 15} \\
\hline CCAA & Cte. (a) & $\mathrm{U}_{\mathrm{t}-1}\left(\beta_{1}\right)$ & $\mathrm{U}_{\mathrm{t}-2}\left(\beta_{2}\right)$ & $R^{2}$ ajustado \\
\hline Andalucía & $-2,51(4,46)$ & $1,12(1,12)$ & & 0,69 \\
\hline Aragón & $1,47(0,72)$ & $0,75(0,13)$ & & 0,61 \\
\hline Asturias & $2,60(1,83)$ & $0,75(0,19)$ & & 0,53 \\
\hline Ast. [2001:4-2004:4] & $4,18(2,11)$ & $0,61(0,21)$ & & 0,43 \\
\hline Baleares & $1,03(1,30)$ & $0,89(0,15)$ & & 0,80 \\
\hline Bal. [2002:1-2004:4] & $3,53(0,49)$ & $0,62(0,06)$ & & 0,82 \\
\hline Canarias & $4,81 \quad(2,24)$ & $0,58(0,20)$ & & 0,34 \\
\hline Cantabria & $4,09(1,91)$ & $0,60(0,19)$ & & 0,28 \\
\hline Castilla y León & $4,32(1,90)$ & $0,60(0,18)$ & & 0,34 \\
\hline Castilla-La Mancha & $3,86(1,54)$ & $0,74(0,16)$ & $-0,14 \quad(0,10)$ & 0,29 \\
\hline C-LM [2001:3-2004:4] & $6,20(1,49)$ & $1,22(0,21)$ & $-0,85(0,26)$ & 0,57 \\
\hline Cataluña & $4,74(2,36)$ & $0,52(0,24)$ & & 0,31 \\
\hline Com. Valenciana & $3,49(1,24)$ & $0,67(0,12)$ & & 0,57 \\
\hline Extremadura & $8,28(5,35)$ & $0,52(0,30)$ & & 0,25 \\
\hline Ext. [2002:2-2004:4] & $8,30(4,33)$ & $0,53(0,24)$ & & 0,23 \\
\hline Galicia & $3,23(2,60)$ & $0,75(0,20)$ & & 0,55 \\
\hline Gal. [2002:1-2004:4] & $6,31 \quad(2,66)$ & $0,52 \quad(0,21)$ & & 0,37 \\
\hline Madrid & $5,70(1,21)$ & $0,01 \quad(0,20)$ & $0,19(0,08)$ & 0,14 \\
\hline Murcia & $8,80(3,29)$ & $0,19(0,31)$ & & - \\
\hline Navarra & $2,68(1,13)$ & $0,51 \quad(0,20)$ & & 0,19 \\
\hline País Vasco & $7,34 \quad(0,99)$ & $0,23(0,10)$ & & - \\
\hline La Rioja & $2,09(1,01)$ & $0,64 \quad(0,18)$ & & 0,38 \\
\hline \multicolumn{5}{|c|}{$\begin{array}{l}\text { NotA: Los errores estándar aparecen entre paréntesis junto al parámetro y son } \\
\text { consistentes con la heterocedasticidad. Se ha contrastado la existencia de } \\
\text { autocorrelación mediante el test de Breusch y Godfrey, basado en el multiplicador de } \\
\text { Lagrange, y en caso de detectar la existencia de autocorrelación se han introducido } \\
\text { como regresores más retardos de la variable endógena y se ha calculado la significación } \\
\text { conjunta de los parámetros } 8 \text { mediante el test de Wald. }\end{array}$} \\
\hline
\end{tabular}




\section{Cuadro 3: Tasa de paro de equilibrio a largo plazo 2001-2004}

\begin{tabular}{lc}
\hline Aragón & 5,93 \\
Asturias [2001:4-2004:4] & 10,63 \\
Baleares [2002:1-2004:4] & 9,37 \\
Canarias & 11,44 \\
Cantabria & 10,14 \\
Castilla y León & 10,68 \\
Castilla-La Mancha [2001:3-2004:4] & 9,70 \\
Cataluña & 9,79 \\
Com. Valenciana & 10,74 \\
Extremadura [2002:2-2004:4] & 17,66 \\
Galicia [2002:1-2004:4] & 13,03 \\
Madrid & 7,07 \\
Navarra & 5,42 \\
País Vasco & 9,55 \\
La Rioja & 5,95 \\
\hline
\end{tabular}

Fuente: Elaboración propia a partir de los datos del cuadro 3.

\subsection{Método de Elmeskov}

Si los métodos de alisamiento adolecen, normalmente, de un sustrato teórico y el método del equilibrio a largo plazo nos proporciona estimaciones puntuales y constantes del pleno empleo, el enfoque de los salarios corrige simultáneamente ambos problemas. ${ }^{7}$ Así, dado que la tasa de paro de pleno empleo no ha permanecido estable en el tiempo, lo adecuado es estimar una "time-varying" NAWRU (Tasa de Desempleo no Aceleradora de los Salarios). Una metodología bastante extendida es la desarrollada por Elmeskov (1993), ${ }^{8}$ donde se estima una NAWRU bajo el

\footnotetext{
7 Otra alternativa, que no se ha contemplado en este trabajo, es utilizar la metodología de la función de producción, que se emplea para estimar la producción potencial (y su crecimiento) de una economía. Para ello, es necesario evaluar los factores productivos (empleo y capital) a su nivel potencial. Un ingrediente básico -junto al nivel potencial de la tasa de actividad y la población en edad de trabajar- del empleo potencial es la NAIRU (Tasa de Desempleo Compatible con un Nivel Estable de Inflación). Un ejemplo reciente de la aplicación de esta metodología para la economía española es el trabajo de Estrada et al. (2004), donde la NAIRU y la curva de Phillips se estiman de forma conjunta mediante el filtro de Kalman (parámetros "time-varying" -véase también sobre este particular el trabajo de Denis et al., 2002).

8 Método ampliamente utilizado en el marco de la OCDE o el FMI -véase también Ball (1997)-.
} 
supuesto de que los cambios en la inflación salarial (salarios nominales) son proporcionales a la brecha del desempleo. Es decir:

$$
\Delta^{2} w_{t}=-\alpha_{t}\left(U_{t}-U_{t}^{*}\right)
$$

donde $w_{t}$ es el logaritmo neperiano de los salarios nominales, ${ }^{9} U_{t}$ es la tasa de desempleo trimestral desestacionalizada y $U_{t}^{*}$ es la NAWRU. Las dos variables inobservables de esa expresión, $a_{t} \mathrm{y} U_{t}^{*}$, se identifican bajo la restricción de que ambas deben permanecer constantes entre dos observaciones consecutivas.

Una estimación de $\alpha_{t}$ se obtiene para dos periodos consecutivos de la siguiente forma:

$$
\alpha_{t}=-\frac{\Delta^{3} w_{t}}{\Delta U_{t}}
$$

y sustituyendo esta última expresión en la anterior obtenemos la NAWRU $\left(U_{t}^{*}\right)$ :

$$
U_{t}^{*}=U_{t}-\frac{\Delta U_{t}}{\Delta^{3} w_{t}} \Delta^{2} w_{t}
$$

Respecto a la NAWRU obtenida por este procedimiento hay que decir que se trata de un indicador de equilibrio a corto plazo, puesto que representa la tasa de desempleo asociada, en un periodo dado y vinculada a la evolución pasada reciente de las variables objeto de estudio, a un crecimiento salarial constante. ${ }^{10}$

Teniendo en cuenta el importante cambio metodológico de la EPA acaecido en el año 2001 y que en ese mismo año deja de publicarse la Encuesta de Salarios de la Industria y los Servicios, se han dividido las estimaciones de las NAWRUs regionales en dos periodos: 1990-2000 y 2001-2004. ${ }^{11}$ En el primer periodo, se aproxima la inflación salarial por la tasa de variación interanual de los salarios nominales (ganancia/hora trabajada). En el segundo periodo, una vez deja de publicarse la citada

$9 \Delta$ (filtro en diferencias) se refiere a $\Delta^{k}=(1-L)^{k}$.

10 Debido a las características de la forma de obtención descrita, la NAWRU resultante es sumamente variable, de modo que lo habitual es alisar la serie original de la NAWRU estimada (mediante medias móviles, el filtro de Hodrick-Prescott, etc.). En este caso, se han alisado las NAWRUs regionales con el filtro de Hodrick-Prescott.

11 Como es bien sabido, también ha habido otros cambios metodológicos en la EPA dentro del periodo muestral (por ejemplo, en 1992 y 1999). Sin embargo, hay que tener en cuenta que en 2001 el cambio de la EPA coincide, como ya se ha mencionado, con que deja de publicarse la Encuesta de Salarios de la Industria y los Servicios. Por ello, se ha optado por presentar las estimaciones en los dos subperiodos correspondientes. Por lo demás, se sigue utilizando la serie homogénea de la EPA (1990-2004) elaborada por el INE. 
encuesta, se aproxima por la tasa de variación interanual del coste salarial total por trabajador (Índice de Coste Laboral 2001-2003 y Encuesta Trimestral de Coste Laboral 2004). En ambos periodos, se utiliza como tasa de desempleo los datos trimestrales desestacionalizados de la EPA (serie homogénea).

Los cuadros 4 y 5 recogen las NAWRUs de las regiones españolas en los periodos 1990-2000 y 2001-2004, respectivamente. ${ }^{12}$ Los comentarios de estos cuadros se presentan a continuación en las reflexiones finales.

\section{Cuadro 4: Medias anuales de las Nawrus trimestrales estimadas para las regiones españolas (1990-2000). Método de Elmeskov}

\begin{tabular}{lccccccccccc}
\hline & 90 & 91 & 92 & 93 & 94 & 95 & 96 & 97 & 98 & 99 & 00 \\
\hline España (*) & 16,2 & 16,3 & 18,3 & 22,6 & 24,1 & 22,9 & 22,1 & 20,6 & 19,6 & 15,7 & 13,9 \\
\hline Andalucía & 26,2 & 27,8 & 29,8 & 31,6 & 32,8 & 33,4 & 33,1 & 32,0 & 30,4 & 28,4 & 26,1 \\
Aragón & 8,5 & 10,1 & 12,0 & 13,8 & 15,0 & 15,4 & 15,1 & 13,9 & 12,2 & 10,2 & 8,2 \\
Asturias & 17,1 & 17,8 & 18,9 & 20,1 & 21,0 & 21,3 & 21,2 & 20,8 & 20,0 & 19,0 & 17,9 \\
Baleares & 10,3 & 11,1 & 12,2 & 13,3 & 14,0 & 14,2 & 13,7 & 12,6 & 11,2 & 9,4 & 7,6 \\
Canarias & 23,9 & 24,6 & 25,4 & 25,8 & 25,6 & 24,6 & 22,9 & 20,7 & 18,4 & 16,0 & 13,8 \\
Cantabria & 15,9 & 17,1 & 18,7 & 20,2 & 21,5 & 22,2 & 22,1 & 21,1 & 19,2 & 16,9 & 14,4 \\
Cataluña & 13,2 & 14,2 & 15,7 & 13,4 & 18,7 & 19,3 & 18,8 & 17,5 & 15,5 & 13,1 & 10,6 \\
Castilla y León & 15,4 & 16,6 & 18,2 & 19,6 & 20,6 & 20,8 & 20,3 & 19,3 & 17,7 & 15,7 & 13,4 \\
Castilla-LM & 12,4 & 13,5 & 15,1 & 16,7 & 18,0 & 18,8 & 18,9 & 18,5 & 17,5 & 16,1 & 14,3 \\
Extremadura & 24,0 & 24,9 & 26,1 & 27,3 & 28,5 & 29,3 & 29,5 & 28,9 & 27,9 & 26,6 & 25,2 \\
Galicia & 11,8 & 12,9 & 14,4 & 15,9 & 17,1 & 17,7 & 17,9 & 17,7 & 17,3 & 16,6 & 15,9 \\
C. Madrid & 11,2 & 13,1 & 15,5 & 17,8 & 19,3 & 19,9 & 19,5 & 18,2 & 16,3 & 14,1 & 11,8 \\
Murcia & 16,7 & 18,6 & 21,1 & 23,2 & 24,5 & 24,8 & 23,8 & 21,8 & 19,1 & 15,9 & 12,5 \\
Navarra & 11,5 & 11,9 & 12,5 & 13,0 & 13,3 & 13,1 & 12,5 & 11,6 & 10,5 & 9,5 & 8,5 \\
País Vasco & 18,5 & 19,6 & 21,0 & 21,3 & 23,1 & 23,2 & 22,5 & 21,1 & 18,9 & 16,1 & 12,9 \\
La Rioja & 8,7 & 10,2 & 12,2 & 13,9 & 15,2 & 15,7 & 15,2 & 14,0 & 12,4 & 10,6 & 8,7 \\
C. Valenciana & 14,6 & 16,3 & 18,6 & 20,6 & 22,0 & 22,4 & 21,8 & 20,2 & 18,0 & 15,5 & 12,9 \\
\hline
\end{tabular}

Fuente: Elaboración propia a partir de los datos homogéneos de la EPA y la Encuesta de Salarios en la Industria y los Servicios (INE).

(*) Tasa de paro observada

\footnotetext{
$12 \mathrm{El}$ filtro se aplica a las series trimestrales y posteriormente calculamos la media anual.
} 
Cuadro 5: Medias anuales de las Nawrus trimestrales estimadas para las regiones españolas (2001-2004). Método de Elmeskov

\begin{tabular}{lcccc}
\hline & 2001 & 2002 & 2003 & 2004 \\
\hline España (*) & 10,6 & 11,5 & 11,5 & 11,0 \\
\hline Andalucía & 19,5 & 19,1 & 18,5 & 17,9 \\
Aragón & 5,5 & 5,6 & 5,8 & 5,9 \\
Asturias & 8,0 & 8,7 & 9,8 & 10,7 \\
Baleares & 5,6 & 7,0 & 8,8 & 10,4 \\
Canarias & 10,9 & 11,2 & 11,4 & 11,9 \\
Cantabria & 8,4 & 9,1 & 10,0 & 10,8 \\
Cataluña & 8,7 & 9,0 & 10,5 & 9,8 \\
Castilla y León & 10,1 & 10,4 & 10,7 & 10,8 \\
Castilla-LM & 8,9 & 9,5 & 9,8 & 9,4 \\
Extremadura & 14,3 & 18,1 & 15,2 & 17,1 \\
Galicia & 10,7 & 15,1 & 12,5 & 13,9 \\
C. Madrid & 7,5 & 6,9 & 7,3 & 6,7 \\
Murcia & 10,4 & 11,0 & 11,6 & 10,6 \\
Navarra & 4,3 & 5,7 & 5,6 & 5,9 \\
País Vasco & 9,5 & 9,7 & 9,4 & 9,6 \\
La Rioja & 4,2 & 7,1 & 6,7 & 6,3 \\
C. Valenciana & 9,6 & 10,7 & 10,9 & 11,2 \\
\hline
\end{tabular}

Fuente: Elaboración propia a partir de los datos homogéneos de la EPA, del Índice de Coste Laboral (hasta 2003:4) y la Encuesta Trimestral de Coste Laboral-2004 (INE).

(*) Tasa de paro observada

\section{Reflexiones finales}

El concepto de pleno empleo resulta bastante ambiguo, lo que se deriva de su carácter de inobservable. En este trabajo se han ofrecido diversas estimaciones del mismo a partir de la tasa de paro de equilibrio de las regiones españoles.

En el periodo 1989-2000, se aprecia una elevada inestabilidad de las tasas de paro, iniciándose en 1995 un proceso sostenido y generalizado de descenso del desempleo que dura hasta final de este periodo. El perfil común en la evolución de las distintas tasas de paro regionales de equilibrio indica que los diferenciales regionales de desempleo no se han reducido en el periodo estudiado y que, por tanto, hay diferenciales de equilibrio. Además, la estimación de la tasa de paro de equilibrio mediante el método de Elmeskov revela la existencia de una apreciable histéresis 
(elevada correlación entre el desempleo observado ${ }^{13}$ y las NAWRUs estimadas).

En el periodo 2001-2004, las tasas de paro se estabilizan y las diferencias interregionales persisten, como evidencian los resultados de la tasa de paro a largo plazo de las regiones españolas (véase el cuadro 3).

Con carácter general podemos subrayar tres hechos: 1) En primer lugar, la existencia de importantes diferencias de equilibrio entre las Comunidades Autónomas, fenómeno que explica la disparidad de las tasas de paro regionales; 2) En segundo lugar, la simetría en su evolución, explicaría la persistencia de tales diferencias; 3) En tercer lugar, la existencia de tres grupos de regiones bien diferenciados. El primero, formado por Aragón, la Comunidad de Madrid, Navarra y la Rioja se encuentran en una situación próxima al pleno empleo. Un segundo grupo, constituido por Andalucía y Extremadura, con tasas de paro de equilibrio superiores al $15 \%$ lo que las sitúa muy alejadas del pleno empleo. Un último colectivo, formado por el resto de Comunidades Autónomas, que tienen tasas de paro de equilibrio próximas al 10\% y en torno a la media nacional.

Como posibles extensiones del análisis desarrollado en este trabajo se pueden apuntar las dos siguientes: a) Sería interesante aplicar la metodología de la función de producción para estimar el desempleo de equilibrio de las regiones españolas; y b) Otra extensión natural del trabajo sería diseccionar los determinantes del desempleo de equilibrio de cada una dichas regiones.

\section{Bibliografía}

Ball, L. (1997): "Disinflation and the NAIRU”, en Romer C.D. y Romer, D.H. (Eds.) (1997): Reducing Inflation: Motivation and Strategy, University of Chicago Press, Chicago, págs. 167-192.

Beveridge, W.H. (1944): Full Employment in a Free Society, George Allen and Unwin, London.

Denis, C., McMorrow, K., y Roeger, W. (2002): "Production Function Approach to Calculating Potential Growth and Output Gaps: Estimates for the EU Member States and the US, European Economy", European

\footnotetext{
${ }^{13}$ En los cuadros 4 y 5 se recoge, en la primera fila, la evolución de la tasa de paro observada en España (que, como sabemos está muy correlacionada con las tasas de paro regionales).
} 
Commission, Economic Papers, 176.

Elmeskov, J. (1993): "High and Persistent Unemployment: Assessment of the Problem and Its Causes", OECD Economics Department Working Papers, 132.

Estrada, A., Hernández de Cos, P. y Jareño, J. (2004): "Una Estimación del Crecimiento Potencial de la Economía Española", Documentos Ocasionales del Banco de España, 0405.

Friedman, M. (1968): "The Role of Monetary Policy", American Economic Review, 58, pp. 1-17.

Keynes, J.M. (1936): The General Theory of Employment, Interest and Money, Macmillan, London.

Pehkonen, J. y Tervo, H. (1998): "Persistence and Turnover in Regional Unemployment Disparities", Regional Studies, 32, pp. 445-458.

Richardson, P., Boone, L., Giorno, C., Meacci, M., Rae, D. y Turner, D. (2000): "The Concept, Policy Use and Measurement of Structural Unemployment: Estimating a Time Varying NAIRU Across 21 OECD Countries", OECD Economics Department Working Papers, 250.

Rogerson, R. (1997): "Theory Ahead of Language in the Economics of Unemployment", Journal of Economic Perspectives, 11, pp. 73-92.

Staiger, D., Stock, J.H. y Watson, M.W. (1997): "How Precise are Estimates of the Natural Rate of Unemployment?", en Romer, C.D. y Romer, D.H. (eds.) (1997): Reducing Inflation: Motivation and Strategy, Chicago, University of Chicago Press, pp. 195-246.

Staiger, D., Stock, J.H. y Watson, M.W. (2001): "Prices, Wages and the U.S. NAIRU in the 1990s", NBER Working Papers, 8320. 